\title{
Evaluation of the Iconic Pain Assessment Tool by a heterogeneous group of people in pain
}

\author{
Chitra Lalloo BHSc, James L Henry PhD
}

C Lalloo, JL Henry. Evaluation of the Iconic Pain Assessment Tool by a heterogeneous group of people in pain. Pain Res Manage 2011;16(1):13-18.

The Iconic Pain Assessment Tool (IPAT) is a novel web-based instrument for the self-report of pain quality, intensity and location in the form of a permanent diary. Originally designed for people with central poststroke pain, the tool is being adapted for a larger, more diverse patient population. The present study aimed to collect evaluative feedback on the IPAT from a heterogeneous sample of individuals with chronic pain. The specific study aims were to evaluate participant comfort with the tool including enjoyment, ease of use and comfort with the electronic medium; to assess perceived value of the tool for communicating pain quality, intensity and location; to gauge participant intent to share their pain diaries with others and use the tool on a regular basis to track their pain over time; to assess the perceived descriptiveness of current IPAT icons and the numerical rating scale; and to identify strengths and weaknesses of the tool to refine the existing prototype.

Written and verbal feedback from individuals with a variety of chronic pain conditions $(n=23)$ were collected in the context of these objectives. Overall, the IPAT was positively endorsed by this heterogeneous sample of people in pain. The authors concluded that the IPAT is a user-friendly instrument that has the potential to help people express, document and share their personal experience with chronic pain.

Key Words: Chronic pain; Consumer driven; Pain diary; Pain self-assessment; Self-report; Web-based instrument

\section{Tool development}

The Iconic Pain Assessment Tool (IPAT) is a novel web-based instrument for the self-report of pain quality, intensity and location in the form of a permanent diary (1). The tool originated as a collaborative graduate project involving the Biomedical Communications program at the University of Toronto (Toronto, Ontario) and the Faculty of Health Sciences at McMaster University (Hamilton, Ontario). The focus of this project was an exploration of pain visualization among individuals with central poststroke pain (CPSP), a relatively rare type of central neuropathic pain (2). Specifically, Émilie McMahonLacharité and author JLH sought to design an interactive, web-based learning module to teach patients with CPSP about the etiology of their condition.

This module was intended to supplement the traditional flow of information from the clinician to the patient. Stemming from this idea of doctor-patient communication came the realization that the exchange of information should ideally be reciprocal because it is the patients themselves who may be viewed as 'experts' in the context of pain experience. Indeed, the importance of patient self-report of pain symptoms is widely recognized $(3,4)$. Therefore, the CPSP educational module was expanded to include a simple instrument to facilitate the translation of patient experience into a visual record that could be rapidly interpreted by health care professionals, clinical researchers and members of the patient's social network. Specifically, the IPAT

\section{L'appréciation de l'outil d'évaluation de la douleur par icônes par un groupe hétérogène de personnes éprouvant des douleurs}

L'outil d'évaluation de la douleur par icônes (OÉDI) est un nouvel instrument Web pour autodéclarer la qualité, l'intensité et le foyer de la douleur sous forme de journal permanent. Conçu au départ pour les personnes ayant des douleurs centrales après un accident vasculaire cérébral, l'outil est adapté pour une population de patients plus large et plus diversifiée. La présente étude visait à colliger des commentaires évaluatifs sur l'OÉDI auprès d'un échantillon hétérogène de personnes éprouvant des douleurs chroniques. Les objectifs précis de l'étude consistaient à évaluer la facilité d'usage de l'outil par le participant, y compris le plaisir, la facilité et l'aisance d'utilisation de l'outil électronique, à évaluer la valeur perçue de l'outil pour communiquer la qualité, l'intensité et le foyer de la douleur, à sonder l'intention du participant de partager son journal de douleur avec d'autres et d'utiliser l'outil régulièrement afin de suivre la douleur au fil du temps, à évaluer la perception du caractère descriptif des icônes et de l'échelle d'évaluation numérique de l'OÉDI et à déterminer les forces et les faiblesses de l'outil en vue d'améliorer le prototype actuel.

Les chercheurs ont colligé les commentaires écrits et verbaux de personnes ayant divers troubles de douleur chronique $(n=23)$, compte tenu de ces objectifs. Dans l'ensemble, cet échantillon hétérogène de personnes éprouvant des douleurs a bien accueilli l'OÉDI. Les auteurs ont conclu que l'OÉDI est un instrument convivial qui a le potentiel d'aider les personnes à exprimer, à consigner et à partager leur expérience personnelle de douleur chronique.

was designed to visually communicate what the pain feels like (quality), how severe it is (intensity) and where it hurts (location) (Figure 1).

The importance of these pain parameters has been recognized as a core domain of the Initiative on Methods, Measurement, and Pain Assessment in Clinical Trials (IMMPACT) group (5). The IPAT certainly does not exist in isolation, but rather represents a further innovation in the decades of work exploring the assessment of pain. The following paragraphs will briefly outline some existing methods of assessing pain quality, intensity and location in relation to the IPAT as well as describe the advantages associated with electronic administration of health scales. Interested readers are encouraged to consult relevant chapters of the Handbook of Pain Assessment for further details (3). Once this foundation has been established, we will describe our intention to expand the IPAT target audience from CPSP to a larger and more diverse pain population.

\begin{abstract}
Assessment of pain quality
As eloquently described by Wagstaff et al (6), “...to communicate adequately what is perceived to another requires possession of a spontaneous vocabulary sufficient to translate feelings into words". Pioneers of pain measurement, Melzack and Torgerson (7), successfully compiled a series of adjectives to describe the various "patterns, colours, and textures" of the pain experience. The sensory component of the
\end{abstract}




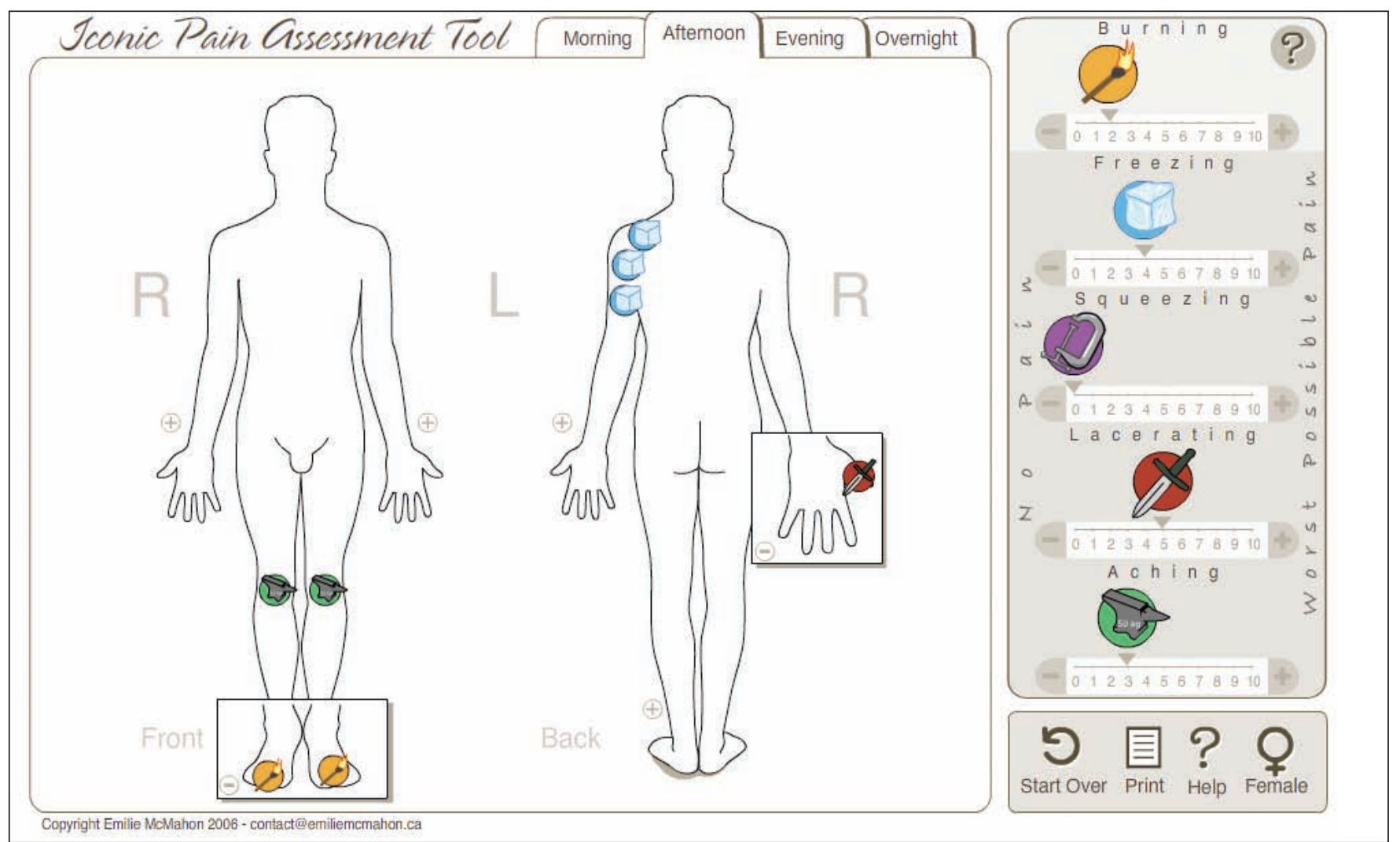

Figure 1) Current iteration of the Iconic Pain Assessment Tool (available at www.emiliemcmahon.ca/pain-tool.html). Reproduced with permission from Émilie McMahon-Lacharité

resulting McGill Pain Questionnaire (MPQ) (8) includes 54 pain adjectives organized into 20 discrete categories and ranked according to implied intensity. For example, the descriptors of the 'temporal' category, in order of increasing intensity, are the following: flickering, quivering, pulsing, throbbing, beating and pounding. Patients are asked to choose the one word from each category that best describes their pain and a total score is then calculated. The subsequently developed Short-Form MPQ (SF-MPQ) includes 11 sensory descriptors from the original scale and is useful for situations requiring a rapid symptom assessment (9). Recently, Dworkin et al (10) developed a new version of the questionnaire (SF-MPQ-2) that includes descriptors for both neuropathic and non-neuropathic pain. Although these instruments can be used to produce a comprehensive and precise explanation of what pain feels like, they also require a fairly advanced degree of literacy in the patient. Thus, the purely text-based medium presents an issue for individuals with limited written or verbal communication skills (11), or a preference for visual communication.

There are existing alternatives to a purely text-based description of pain quality. Swanston et al (12), recognizing a need for reduced reliance on the linguistic competence of patients, developed interactive computer-generated animations to represent various types of pain. This scale includes interactive animations for the qualities of pressure, burning, throbbing and piercing pain. The photographer Deborah Padfield described another interesting example of pain visualization (13). Using the technique of photomontage, Padfield worked with chronic pain patients to help them create striking and evocative visual depictions of their pain. Patients reported that this exercise helped to create an 'emotional outlet' for their suffering, and physicians stated that the resulting imagery allowed them to gain a better understanding of the nature of chronic pain.

Another viable alternative to purely text-and verbal-based protocols is the careful fusion of imagery and words. Specifically, the use of stylized graphic images (termed 'icons') can minimize native-language and language-level barriers (14), which may help to 'level the field' in terms of description of pain.

The IPAT was designed to capitalize on these potential advantages of icon-based communication to help patients better describe their experiences. The IPAT features icons for five pain qualities (burning, freezing, squeezing, lacerating and aching) that were selected based on prevalence in the CPSP literature and consumer consultation. Émilie McMahon-Lacharité created a visual metaphor for each icon based on an image search using resources such as the Internet, magazines, television commercials and comic book depictions of pain (1). The current visual metaphors included in the IPAT are a flame on a matchstick (burning pain), an ice cube (freezing pain), a vice (squeezing pain), a knife (lacerating pain) and an anvil (aching pain). Patients choose among these icons to describe their current pain sensations. To our knowledge, the IPAT is the only web-based instrument that uses iconography in the description of pain quality.

\section{Assessment of pain intensity}

A well-known measure of pain intensity is the visual analogue scale (VAS), which consists of a horizontal or vertical line of fixed length with anchors such as 'no pain' and 'worst pain imaginable' (15). The patient is asked to place a mark along the line to directly estimate the magnitude of their pain. Originally called the 'graphic rating method' (16), the VAS has since been adapted into numerous formats and used extensively in the measurement of pain intensity $(15,17)$. Interestingly, while the VAS appears to provide a continuous measure of pain intensity, evidence suggests that respondents tend to divide the VAS line into smaller increments of 5 or 10 , essentially treating it as an 11- or 21-point scale (18).

Another form of intensity assessment is focused on the development of scales that minimize cognitive demands on the patient. For example, concrete ordinal rating scales, such as the Pieces of Hurt tool (19), use physical objects (eg, poker chips) to represent different amounts of pain. Meanwhile, the Wong-Baker Faces Pain Scale 
depicts the spectrum of pain through a series of sexually and ethnically neutral hand-drawn faces (20). Patients are asked to examine each face and then select the one that best describes their current pain. This type of scale has been successfully applied to pediatric populations as well as adults with cognitive disorders $(21,22)$.

The MPQ uses a numerical rating scale (NRS) to quantify pain intensity, in which patients are asked to rate the severity of each pain quality as $0=$ none, $1=$ mild, $2=$ moderate or $3=$ severe. The subsequently developed SF-MPQ, SF-MPQ-2 and Brief Pain Inventory $(23,24)$ all feature an 11-point NRS ranging from 0 to 10 . Indeed, the IMMPACT group recommends the use of this type of NRS to measure pain intensity (4).

The IPAT features an NRS ranging from 0 ('no pain') to 10 ('worst possible pain') below each pain quality icon. By clicking on terminal buttons, users can easily assign an intensity rating to each pain quality.

\section{Assessment of pain location}

The distribution of pain across body regions is a critical component of pain assessment. Simple diagrams of the anterior and posterior aspects of the body are commonly used in this type of assessment. In some cases, patients are instructed to shade the regions where they feel pain and then a transparent template is placed over the diagram to generate a score $(25-27)$. Certain computer-based assessment scales allow patients to create 'dynamic pain drawings' using a mouse on a fixed body template (28).

The IPAT interface allows users to select relevant pain qualities, assign an intensity rating, and then drag and drop small circular pain icons onto a simple body map to indicate location. The user also has the option of expanding the hands and feet to more precisely document the location of pain among the digits.

Thus, the IPAT is uniquely positioned to facilitate the self-report of pain quality through a mixture of iconography and descriptors, pain intensity of each quality via NRS, and pain location by the spatial arrangement of icons on a simple body map.

\section{Method of administration}

Although the earliest pain assessment tools were necessarily paper based, there has been a recent shift toward electronic administration of pain scales (28-30). Advantages of this approach over paper-based techniques include minimization of errors in data transfer and transcription, ability to capture time-stamped data, ease of data sharing, increased compliance and heightened patient satisfaction (30-33).

The IPAT was developed in Adobe Flash (Adobe Systems Inc, USA) and is freely available online (www.emiliemcmahon.ca/ pain-tool.html). Users can access the tool from any computer or mobile device with Flash capability to document their pain parameters in real time. After documenting their pain, users can print a hard copy of their pain diary or save a copy as a PDF file. Regular use of the IPAT facilitates the creation of a permanent record (collection of PDF files) of pain quality, intensity and location over time.

\section{Expanding the target audience for the IPAT}

As described above, the IPAT was originally designed for the target population of individuals with CPSP. However, because the unique features of the tool have the potential to benefit other groups of people in pain, the decision was made to adapt the IPAT for a larger and more diverse audience. The first step in this process of adaptation was to determine whether the current iteration of the IPAT was palatable to potential future users. Therefore, the present study collected evaluative feedback on the IPAT from a heterogeneous sample of individuals with chronic pain.

Our specific aims were to evaluate participant comfort with the tool including enjoyment, ease of use and comfort with the electronic medium; to assess perceived value of the tool for communicating pain quality, intensity and location; to gauge participant intent to share their pain diaries with others and use the tool on a regular basis to
TABLE 1

Types of chronic pain reported by study participants

\begin{tabular}{ll}
\hline Arthritis $(n=3)$ & Low back pain $(n=1)$ \\
Bursitis $(n=1)$ & Nerve damage $(n=1)$ \\
Bulging disc $(n=1)$ & Osteoarthritis $(n=2)$ \\
Diverticulum $(n=1)$ & Polymyalgia rheumatica $(n=2)$ \\
Fibromyalgia $(n=3)$ & Rheumatoid arthritis $(n=3)$ \\
Herniated vertebrae $(n=2)$ & Sciatica $(n=2)$ \\
Joint trauma $(n=1)$ & Shoulder pain $(n=1)$ \\
Knee pain $(n=1)$ & Subluxated ribs $(n=1)$ \\
\hline
\end{tabular}

Some participants $(n=23)$ reported multiple types of chronic pain

track their pain over time; to assess the perceived descriptiveness of the current IPAT icons and NRS; and to identify strengths and weaknesses of the tool to refine the existing prototype.

\section{METHODS}

This project was approved by the Hamilton Health Sciences/ McMaster Health Sciences Research Ethics Board (REB \#07-339), and all participants provided free and informed written consent. No personal or identifying information was collected from study participants.

From December 2008 to March 2009, an evaluation of the IPAT was conducted in a diverse group of individuals with chronic pain. The study involved 23 participants and was conducted in an informal setting.

\section{Inclusion criteria}

To be eligible for the present study, participants were required to be at least 18 years of age, exhibit stable, nondeteriorating health and be currently experiencing chronic pain of any origin. Participants also needed to be deemed capable of giving free and informed consent, and be able to read, write and speak English.

\section{Study protocol}

After informed consent was documented, each participant was given a short demonstration (approximately $5 \mathrm{~min}$ ) of tool functionality using the investigators' laptop computer and external mouse. During this demonstration, author CL used a standard guide to explain the ability to describe different types of pain, assign an individualized intensity rating and show pain location. Subsequently, study participants used the tool to document their own pain experience. Participants were asked to 'think aloud' as they navigated the tool, providing a source of immediate, minimally filtered qualitative feedback on the IPAT. The outcome measures used by the investigators were a written questionnaire developed specifically for the present study, a discussion between participants and CL about the tool, and investigator observation of the participant as they navigated the tool. The accumulated feedback was used to address the aims of testing.

Data collected from the written questionnaire were summarized by descriptive statistics such as arithmetic means \pm SDs to describe the central tendency and data dispersion, respectively. Qualitative data in the form of written and verbal feedback are presented as direct quotations from study participants (identifiers removed).

\section{RESULTS}

\section{Study participants}

The study sample was drawn from a local chronic pain support group that meets on a voluntary, monthly basis in Burlington, Ontario, as well as through word-of-mouth recommendations within the Hamilton community. As shown in Table 1, these individuals exhibited a variety of chronic pain syndromes, providing a diverse sample in which to evaluate the IPAT.

The average severity of pain experienced by these participants on most days, according to self-report, was 5.2 on an 11-point NRS. This 


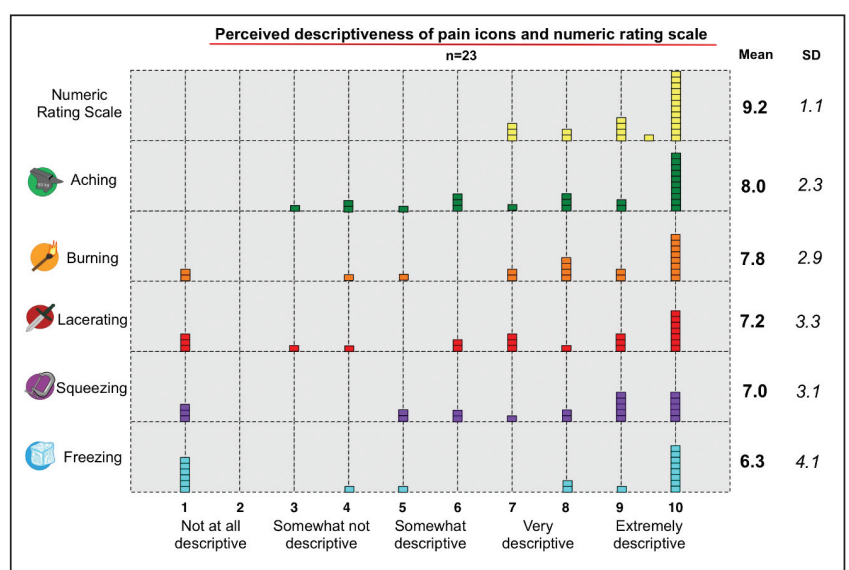

Figure 2) Perceived descriptiveness of the Iconic Pain Assessment Tool icons and numerical rating scale in relation to the quality and intensity of chronic pain, respectively. Frequency distribution of responses and arithmetic means \pm SDs are shown for 23 subjects. Note for interpretation: Each individual block represents a single participant response. For instance, a total of three participants gave the numerical rating scale a rating of 7

finding is roughly comparable to the average intensity of 6.3 reported by a national chronic pain survey involving 340 respondents (34).

\section{Participant comfort with the IPAT}

Participants were asked to rate the tool on a 10-point NRS in terms of enjoyment and ease of use, as well as their personal level of comfort with the electronic medium. The scale anchors for these items were, respectively, "didn't like it at all/liked it very much", "not easy at all/ extremely easy" and "not comfortable at all/extremely comfortable". Overall, participants liked using the tool (mean 8.4 \pm 1.6 ), found it easy to navigate (mean $8.3 \pm 1.9$ ) and were comfortable with its computerbased nature (mean $7.7 \pm 2.1$ ). Overall, 21/23 participants (91\%) reported that they personally had access to a computer. As well, 22/23 participants (96\%) reported that "...no part of the [interface] was difficult to read or see".

\section{Perceived value of tool for communicating pain sensations}

The literature suggests that individuals with chronic pain often feel profoundly misunderstood by people without chronic pain (35). The participants were presented with the statement, "Other people, such as friends, family and co-workers, have trouble understanding my pain experiences". Level of agreement was assessed by an NRS ranging from 1 ('strongly disagree') to 10 ('strongly agree'). On average, study participants voiced agreement (mean $7.9 \pm 2.4$ ) with this statement.

An intended function of the IPAT is to allow individuals to communicate their pain experience with others. The authors were interested in assessing how much value participants would attribute to the tool in this context. Using a 10-point NRS ranging from 'not valuable at all' to 'extremely valuable', participants gave the tool a mean rating of $8.9 \pm 1.5$ for value in pain communication.

Due to the novel nature of the tool, a likely audience for the pain diaries has yet to be characterized. Therefore, participants were asked to assess the value that the tool would have for "a person who they wish could understand" their chronic pain. The mean response for this item was $7.7 \pm 2.5$.

Other potential audiences for the pain diaries are health care professionals and close family members. Employing a 10-point NRS ranging from "highly unlikely" to "highly likely", participants rated the likelihood that examination of completed pain diaries would help these groups to better understand the nature of their chronic pain. The mean responses for these likelihood items were 8.4 \pm 2.1 and $7.9 \pm 2.4$ for health care professionals and close family members, respectively.
Intent to share pain diaries with others

Closely related to the notion of pain communication is the degree to which participants are willing or likely to share their completed pain diaries with other parties. Using a 10-point NRS ranging from "highly unlikely" to "highly likely", participants reported the likelihood that they would show their pain diaries to various individuals. Overall, participants were highly likely to show their pain diaries to a specialist (mean 9.2 \pm 1.9 ) or family physician (mean 9.0 2.1 ). On average, they were also likely to share this information with "a person who they wish could understand" their pain (mean $8.4 \pm 2.3$ ) and somewhat less likely to share with close family members (mean $7.8 \pm 2.4$ ).

Interestingly, participants were less likely to show their pain diar-

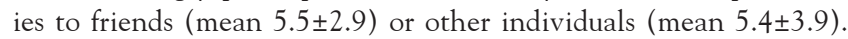
On informal probing for the logic behind these responses, some individuals cited feelings of awkwardness in showing their pain diaries to a friend and a belief that no other individuals would be interested in reading their pain record. In contrast, the responses of other participants indicate that they would share their pain diaries with anyone who wished to see them, suggesting additional recipients such as government adjudicators (agents and representatives) and significant others.

\section{Perceived value of tool for monitoring pain over time}

Another use of the tool is to facilitate creation of a permanent record of pain over time in the form of accumulated PDF pain diaries. Participants were asked to assess the value of the tool for this purpose using a 10-point NRS ranging from "not valuable at all" to "extremely valuable". The mean response for this item was $8.9 \pm 1.3$. The construction of a comprehensive record of pain over time requires regular use of the tool. Given that the tool is currently available on the Internet, participants were asked to rate the likelihood that they would use the tool at least once a month, once a week and once a day. Perhaps reflecting the relatively low power of the present study, these data are inconsistent but may indicate that a diminishing proportion of participants would be willing to use the tool on a monthly (mean $8.3 \pm 2.3$ ), weekly (mean 7.1 \pm 2.9 ) and daily (mean $6.6 \pm 3.4$ ) basis. On probing for the logic underlying the intended frequency of tool use, some individuals stated that the relatively stable nature of their pain would render weekly or daily diaries somewhat redundant. Conversely, participants who experienced frequent changes in their chronic pain seemed more amenable to daily use of the tool. Because these observations are anecdotal, future studies will aim to formally characterize use of the tool.

\section{Evaluation of the IPAT NRS and pain quality icons}

The IPAT allows users to assign a specific intensity on an NRS from 0 to 10 to each relevant pain quality. Participants were asked to rate the degree to which the IPAT NRS and icons described the intensity and quality of their chronic pain. As shown in Figure 2, the IPAT NRS was given universally high ratings, ranging from 7 ('very descriptive') to 10 ('extremely descriptive') with a mean of $9.2 \pm 1.1$.

The most frequent rating for each of the five icons was 10 (extremely descriptive), and the mean ratings ranged from 6.3 to 8.0. The observed dispersion of the data may reflect the heterogeneous nature of the study sample, which reported 16 different types of chronic pain (Table 1). It is important to note that, unlike other outcome measures, wide data dispersion is a desirable characteristic for these ratings. If all icons received uniformly high ratings of descriptiveness, this would suggest that separate pain quality descriptors do not provide more information than a unifactorial intensity rating.

\section{Direct participant feedback}

In addition to the quantitative data yielded from the NRSs, the authors also wished to capture the ideas and opinions of study participants 'in their own words'. A sample of such feedback is presented to consolidate the themes addressed earlier: 
I feel that this tool will prove to be very useful, hopefully helping general practitioners to understand the degree of pain that their patients are trying to convey.

It is great to have an icon to use to describe the particular type of pain one is experiencing.

Visual, instant feedback simplified.

Pictures are a good idea, especially if dealing with someone whose first language is not English.

Again, it is a very clear, concrete way to show the doctor and helps with the memory - or lack of it.

I would like to get at this on the Internet as soon as possible.

When my hands are tight or stiff and hurting...[using the tool] would present some problems.

Because pain can come and go with different intensity it is hard to show this on the program.

[Using the tool] would make me think about my pain, but I would rather try and forget about it.

Unless you live with it, then [you can't] understand.

\section{Participant comfort with the IPAT}

\section{DISCUSSION}

On average, the tool was rated as both enjoyable and easy to use, although participants were somewhat less comfortable with its computer-based nature. The majority of current pain assessment tools are paper based, which could be contributing to the moderate comfort participants feel with the electronic nature of the IPAT.

As well, it is possible that only those individuals who felt somewhat comfortable with the computer-based nature of the tool decided to volunteer for the study. However, there is literature-based evidence that chronic pain patients are amenable to electronic information and assessment tools $(30,32,36)$.

\section{Perceived value of tool}

The data indicate that participants view the tool in a positive light with regard to the objectives of communicating pain sensations and tracking pain over time. On probing for ways to improve the tool, participants proposed the addition of new features such as the ability to add text to the pain diaries, graph data longitudinally, store their diaries in a centralized database and control an alarm system to emit regular reminders to use the tool. These suggestions align with the existing literature on real-time data capture, a strategy to minimize recall bias and improve compliance by allowing patients to report symptoms at particular moments in time $(33,37)$. This method has been successfully applied to electronic pain diaries for specific pain populations (38) and could potentially be adapted for the IPAT.

Because the mere availability of systematic pain assessment data is not sufficient to affect clinical decision making (39), we recognize that early clinician involvement in tool development may increase their likelihood of later uptake. Indeed, qualitative studies report that many clinicians wish to be involved in the planning of outcome assessment protocols (40). Therefore, we intend to conduct future studies to assess the perceived value of the IPAT among health care professionals. These data will also be used to determine the most appropriate method of generating a summary score for the instrument.
Intent to share pain diaries with others

Participants were most strongly inclined to share their pain diaries with health professionals, followed by individuals who they wish could understand their pain, and close family members. It is important to realize that these preliminary data are merely reflective of the participants' intended use of the pain diaries and may not necessarily be indicative of actual use. Thus, prospective studies with participant follow-up will be needed to assess individual compliance with the tool.

\section{Descriptiveness of IPAT pain icons}

The current icons illustrate five different pain qualities (burning, freezing, squeezing, lacerating and aching). Given the positive participant response to the idea of expressing pain through iconography, this icon bank will be improved and expanded to include other types of pain. This future work will examine the consistency with which patients negotiate meaning with the pain iconography and assess the need for additional icons within specific pain populations.

\section{Potential benefit for individuals living with chronic pain}

The prevalence of chronic pain ranges from $19 \%$ to $29 \%$ of the general population $(34,41)$. The IPAT could benefit people living with chronic pain in several important ways. First, the web-based nature of the tool affords a high level of accessibility to the average consumer (42). Second, the electronic format of the pain diary facilitates rapid data storage and dissemination in the form of PDF files. Third, the use of icons to depict pain quality creates real-world points of reference and minimizes reliance on the vocabulary of patients. Lastly, and perhaps most significantly, every stage of tool development has benefited from the direct consumer feedback of individuals living with chronic pain. This patient perspective will continue to drive development of the IPAT, which, in combination with education and self-management strategies (43), should allow people living with chronic pain to better monitor and manage their condition. This patient empowerment is particularly important given that pain sufferers are likely to visit a wide range of practitioners over the course of their disease(s) and must often take responsibility for tracking their pain.

\section{CONCLUSIONS}

Overall, the IPAT was positively endorsed by this heterogeneous sample of people in pain. Our conclusion is that the IPAT, originally designed for individuals with CPSP, is a user-friendly instrument that should be further refined for a larger and more diverse pain population.

ACKNOWLEDGEMENTS: CL was funded with an Alexander Graham Bell Canada Graduate Scholarship from the Natural Sciences and Engineering Research Council of Canada. The authors thank Émilie McMahon-Lacharité, who created the IPAT, along with Dr Linda Wilson-Pauwels of the University of Toronto (Biomedical Communications) for continued support and consultation during the development of this study. The authors also thank Dr Jennifer Stinson (University of Toronto) for her generous guidance regarding study design and early versions of the manuscript, as well as Dr Guy Petroz (University of Toronto) for lending expertise on the technical side of the tool's software. CL thanks Dr Kristina Trim, Dr Joy MacDermid and Dr Delsworth Harnish (McMaster University) for providing feedback on early drafts of the manuscript, lending advice on qualitative research methods and participating in her graduate supervisory committee. Bartosz Orzel and Susan Jo provided the initial efforts to obtain approval from the Research Ethics Board and put forth helpful suggestions regarding study design. In particular, members of the Burlington Chronic Pain Advocacy Group and Swami Arundhati's yoga group are gratefully acknowledged for their participation in this study and willingness to share ideas about the tool. Swami Arundhati is also gratefully acknowledged for her enthusiastic recommendation of the tool to students of her yoga classes. 


\section{REFERENCES}

1. McMahon E, Wilson-Pauwels L, Henry JL, Jenkinson J, Sutherland B, Brierley M. The Iconic Pain Assessment Tool: Facilitating the translation of pain sensations and improving patient-physician dialogue. J Biol Commun 2008;34:E20-E24.

2. Henry JL, Lalloo C, Yashpal K. Central poststroke pain: An abstruse outcome. Pain Res Manage 2008;13:41-9.

3. Jensen MP, Karoly P. Self-report scales and procedures for assessing pain in adults. In: Turk DC, Melzack R, eds. Handbook of Pain Assessment. New York: The Guilford Press, 2001:15.

4. Turk DC, Dworkin RH, Burke LB, et al. Developing patient-reported outcome measures for pain clinical trials: IMMPACT recommendations. Pain 2006;125:208-15.

5. Turk DC, Dworkin RH, Allen RR, et al. Core outcome domains for chronic pain clinical trials: IMMPACT recommendations. Pain 2003; 106:337-45

6. Wagstaff S, Smith O, Wood P. Verbal pain descriptors used by patients with arthritis. Ann Rheum Dis 1985;44:262-5.

7. Melzack R, Torgerson WS. On the language of pain. Anesthesiology 1971;34:50-9.

8. Melzack R. The McGill Pain Questionnaire: Major properties and scoring methods. Pain 1975;1:277-99.

9. Melzack R. The short-form McGill Pain Questionnaire. Pain 1987;30:191-7.

10. Dworkin R, Turk DC, Revicki DA, et al. Development and initial validation of an expanded and revised version of the Short-form McGill Pain Questionnaire (SF-MPQ-2). Pain 2009;144:35-42.

11. Craig K. The construct and definition of pain in developmental disability. In: Symons FJ, Oberlander TF, eds. Pain in Individuals with Developmental Disabilities. Baltimore: Brookes, 2006:7-18.

12. Swanston M, Abraham C, Macrae WA, et al. Pain assessment with interactive computer animation. Pain 1993;53:347-51.

13. Padfield D. Perceptions of Pain. Stockport: Dewi Lewis Publishing, 2003.

14. Beardon C. Research into iconic communication at the University of Brighton. Artif Intell Rev 1995;9:91-3.

15. Streiner D, Norman G. Health Measurement Scales: A Practical Guide to their Development and Use. New York: Oxford University Press, 2008:41.

16. Hayes MHS, Patterson DG. Experimental development of the graphic rating method. Psychol Bull 1921;18:98-9.

17. Stinson JN, Kavanagh T, Yamada J, Gill N, Stevens B. Systematic review of the psychometric properties, interpretability and feasibility of self-report pain intensity measures for use in clinical trials in children and adolescents. Pain 2006;125:143-57.

18. Jensen MP, Turner JA, Romano JM. What is the maximum number of levels needed in pain intensity measurement? Pain 1994;58:387-92.

19. Hester NO, Foster RL, Kristensen K. Measurement of pain in children: Generalizability and validity of the pain ladder and poker chip tool. In: Tyler DC, Krane EJ, eds. Advances in Pain Research and Therapy, vol 15: Pediatric Plan. New York: Raven Press, 1990:79-84.

20. Wong D, Baker C. Pain in children: Comparison of assessment scales. Pediatr Nurs 1988;14:9-17.

21. Bieri D, Reeve RA, Champion GD, Addicoat L, Ziegler JB. The Faces Pain Scale for the self-assessment of the severity of pain experienced by children: Development, initial validation, and preliminary investigation for ratio scale properties. Pain 1990;41:139-50.

22. Kim E, Buschmann M. Reliability and validity of the Faces Pain Scale with older adults. Int J Nurs Stud 2006;43:447-56.

23. Daut RL, Cleeland CS, Flanery RC. Development of the Wisconsin Brief Pain Questionnaire to assess pain in cancer and other diseases. Pain 1983;17:197-210.
24. Tan G, Jensen MP, Thornby JI, Shanti BF. Validation of the Brief Pain Inventory for chronic nonmalignant pain. J Pain 2004;5:133-7.

25. Margolis RB, Tait RC, Krause SJ. A rating system for use with patient pain drawings. Pain 1986;24:57-65.

26. Escalante A, Lichtenstein MJ, White K, Rios N, Hazuda HP. A method for scoring the pain map of the McGill Pain Questionnaire for use in epidemiologic studies. Aging (Milano) 1995;7:358-66.

27. Lacey RJ, Lewis M, Jordan K, Jinks C, Sim J. Interrater reliability of scoring of pain drawings in a self-report health survey. Spine 2005;30:E455-8.

28. Jamison RN, Fanciullo GJ, Baird JC. Computerized dynamic assessment of pain: Comparison of chronic pain patients and healthy controls. Pain Med 2004;5:168-77.

29. Peters ML, Sorbi MJ, Kruise DA, Kerssens JJ, Verhaak PF, Bensing JM. Electronic diary assessment of pain, disability and psychological adaptation in patients differing in duration of pain. Pain 2000;84:181-92.

30. Stinson JN, Petroz GC, Tait G, et al. e-Ouch: Usability testing of an electronic chronic pain diary for adolescents with arthritis. Clin J Pain 2006;22:295-305.

31. Stone AA, Broderick JE, Schwartz JE, Shiffman S, Litcher-Kelly L, Calvanese P. Intensive momentary reporting of pain with an electronic diary: Reactivity, compliance, and patient satisfaction. Pain 2003;104:343-51.

32. Stone AA, Shiffman S, Schwartz JE, Broderick JE, Hufford MR. Patient compliance with paper and electronic diaries. Control Clin Trials 2003;24:182-99.

33. Morren M, Dulmen SV, Ouwerkerk J, Bensing J. Compliance with momentary pain measurement using electronic diaries: A systematic review. Eur J Pain 2009;13:354-65.

34. Moulin DE, Clark AJ, Speechley M, Morley-Forster PK. Chronic pain in Canada - prevalence, treatment, impact and the role of opioid analgesia. Pain Res Manage 2003;7:179-84.

35. Jackson J. Chronic pain and the tension between the body as subject and object. In: Csordas TJ, ed. Embodiment and Experience: The Existential Ground of Culture and Self. New York: Cambridge University Press, 2003:201-28.

36. Hochlehnert A, Richter A, Bludau HB, et al. A computer-based information-tool for chronic pain patients. Computerized information to support the process of shared decision-making. Patient Educ Couns 2006;61:92-8.

37. Hufford MR, Shiffman S, Paty J, Stone AA. Ecological momentary assessment: Real-world, real-time measurement of patient experience. In: Fahrenberg J, Myrtek M, eds. Progress in Ambulatory Assessment: Computer-Assisted Psychological and Psychophysiological Methods in Monitoring and Field Studies. Seattle: Hogrefe \& Huber, 2001:69-92.

38. Stinson JN, Stevens BJ, Feldman BM, et al. Construct validity of a multidimensional electronic pain diary for adolescents with arthritis. Pain 2008;136:281-92.

39. Hadjistavropoulos T, MacNab YC, Lints-Martindale A, Martin R, Hadjistavropoulos H. Does routine pain assessment result in better care? Pain Res Manage 2009;14:211-6.

40. Garland AF, Kruse M, Aarons GA. Clinicians and outcome measurement: What's the use? J Behav Health Serv Res 2003;30:393-405.

41. Breivik H, Collett B, Ventafridda V, Cohen R, Gallacher D. Survey of chronic pain in Europe: Prevalence, impact on daily life, and treatment. Eur J Pain 2006;10:287-333.

42. Murray E, Burns J, See TS, Lai R, Nazareth I. Interactive Health Communication Applications for people with chronic disease. Cochrane Database Syst Rev 2005;(4):CD004274.

43. Craig K. The social communication model of pain. Can Psychol 2009;50:22-32. 


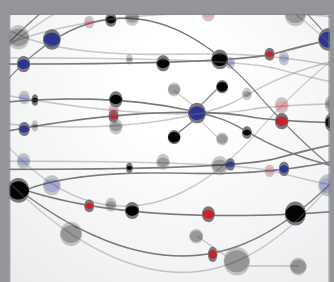

The Scientific World Journal
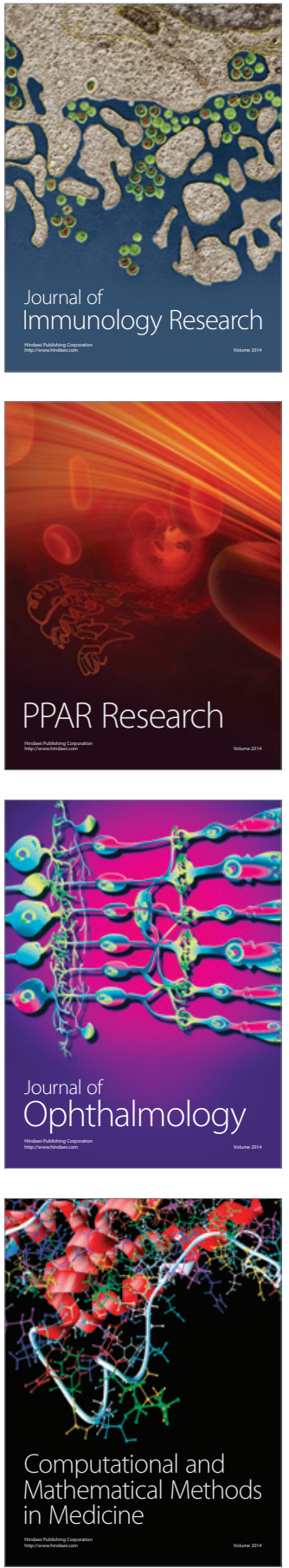

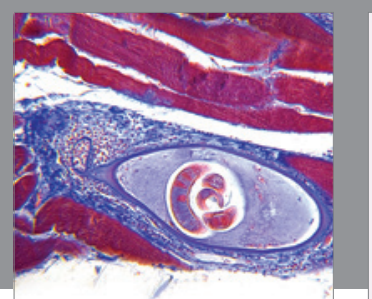

Gastroenterology Research and Practice

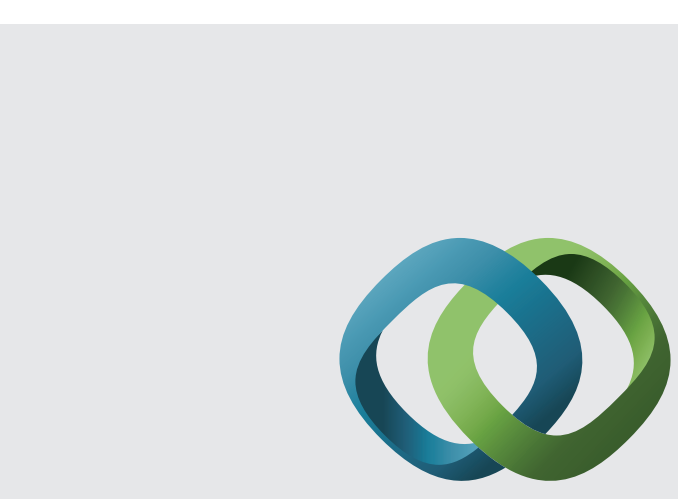

\section{Hindawi}

Submit your manuscripts at

http://www.hindawi.com
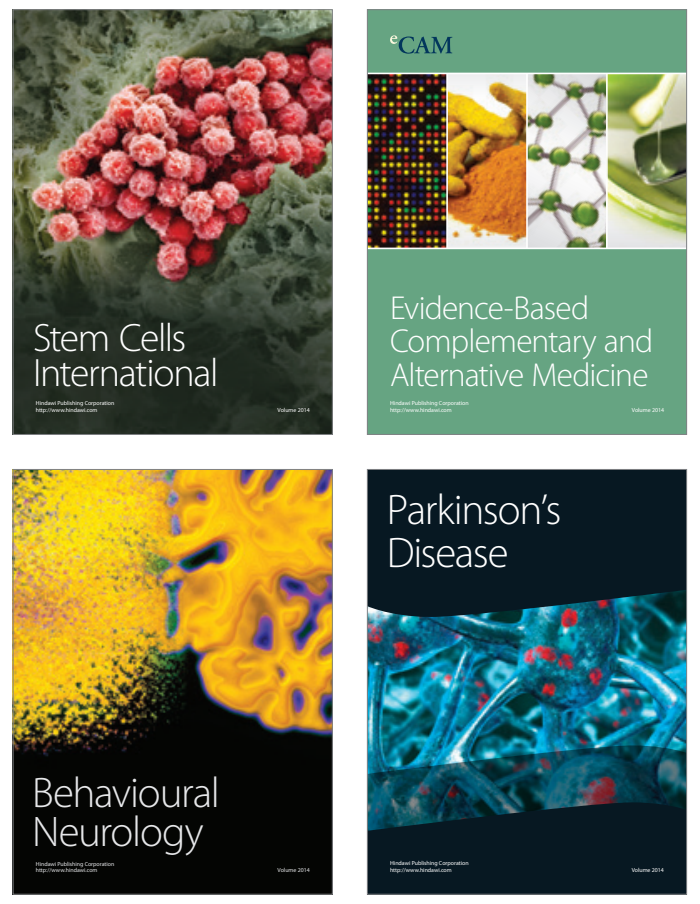
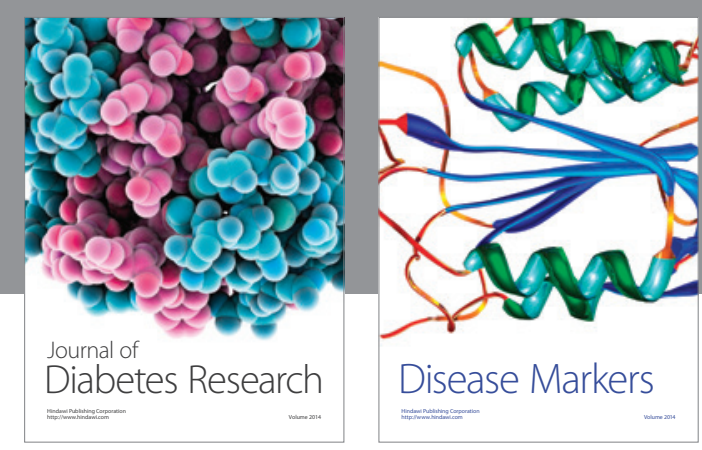

Disease Markers
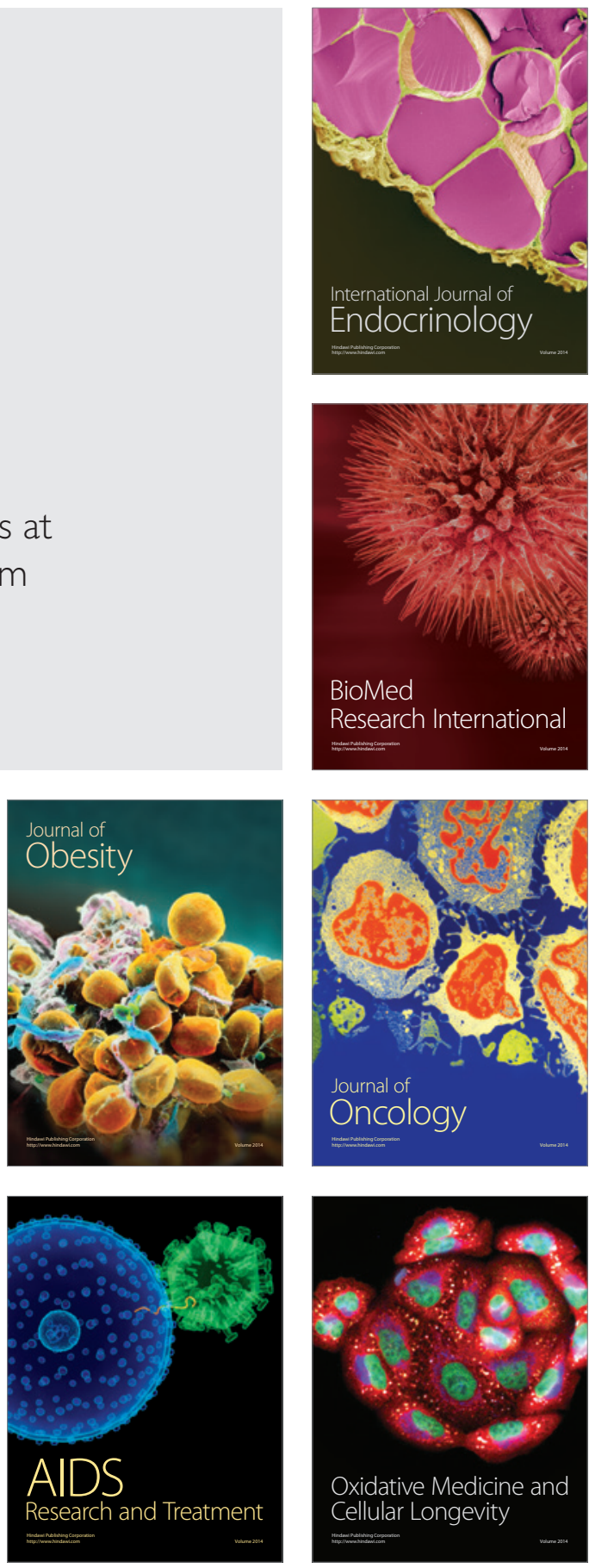